\title{
Multiple mucosa-associated lymphoid tissue organs involving marginal zone B cell lymphoma: organ-specific relationships and the prognostic factors. Consortium for improving survival of lymphoma study
}

\author{
Sung Yong Oh • Won Seog Kim • Jin Seok Kim • Seok Jin Kim • Suee Lee • Dae Ho Lee • Jong-Ho Won • \\ In Gyu Hwang • Min Kyoung Kim • Soon Il Lee • Yee Soo Chae • Deok-Hwan Yang • Hye Jin Kang • \\ Chul Won Choi · Jinny Park • Hyo Jung Kim · Jung Hye Kwon • Ho Sup Lee • Gyeong-Won Lee • \\ Hyeon Seok Eom · Jae-Yong Kwak • Won Sik Lee • Cheolwon Suh • Hyo-Jin Kim
}

Received: 18 July 2010/Revised: 10 August 2010/Accepted: 22 August 2010/Published online: 14 September 2010

(C) The Japanese Society of Hematology 2010

\begin{abstract}
According to a previous review, multiple mucosa-associated lymphoid tissue (MALT)-organs involving marginal zone B cell lymphomas (MZLs) are present in $10-30 \%$ of patients. However, the clinical features and specific relationships among involved organs are yet to be clearly identified. In this study, we conducted retrospective analyses of multiple MALT organs involving MZLs (MM-MZLs) to identify their clinical features,
\end{abstract}

S. Y. Oh · S. Lee $\cdot$ H.-J. Kim $(\bowtie)$

Department of Internal Medicine, Dong-A University College

of Medicine, 3-1 Dongdaeshin-dong, Seo-gu,

Busan 602-715, Korea

e-mail: kimhj@dau.ac.kr

W. S. Kim · S. J. Kim

Division of Hematology/Oncology, Department of Medicine,

Samsung Medical Center, Sungkyunkwan University School

of Medicine, Seoul, Korea

J. S. Kim

Department of Internal Medicine, Yonsei University College

of Medicine, Seoul, Korea

D. H. Lee $\cdot$ C. Suh

Department of Internal Medicine, Asan Medical Center,

University of Ulsan College of Medicine, Seoul, Korea

J.-H. Won

Department of Internal Medicine, Soon Chun Hyang University,

University College of Medicine, Seoul, Korea

I. G. Hwang

Division of Hematology-Oncology, Department of Medicine,

Chung-Ang University Young San Hospital, Seoul, Korea

M. K. Kim

Division of Oncology, Department of Internal Medicine,

Yeungnam University College of Medicine, Taegu, Korea treatment, prognosis, and specific relationships among involved organs. For analysis, between June 1987 and June 2009, a total of 55 patients from 17 different institutions in Korea, all of whom were histologically diagnosed with MM-MZL, were included in this study. MM-MZL was defined as MZL involving more than 2 different MALT organs. Multiple involvements within one MALT organ (e.g. both side ocular lesions, multiple lung nodules, and

S. I. Lee

Department of Internal Medicine, Dankook University

College of Medicine, Cheonan, Korea

Y. S. Chae

Department of Hematology/Oncology, Kyungpook National University School of Medicine, Taegu, Korea

D.-H. Yang

Department of Hematology-Oncology, Chonnam National University Hwasun Hospital, Hwasun, Jeollanam-do, Korea

H. J. Kang

Division of Hematology-Oncology, Department of Internal Medicine, Korea Cancer Center Hospital, Seoul, Korea

C. W. Choi

Division of Oncology-Hematology, Department of Internal Medicine, Korea University Medical Center, Seoul, Korea

J. Park

Department of Internal Medicine, Gachon Medical School, Inchon, Korea

H. J. Kim · J. H. Kwon

Department of Internal Medicine, Hallym University College of Medicine, Seoul, Korea 
multiple stomach lesions, etc.) were excluded from this study. The male/female ratio of the 55 patients was 41/14. The median age of our subjects was 59 years (range 30-82 years). MM-MZL without lymph node (LN) was detected only in 9 patients (36.2\%). Bone marrow (BM) involvement was observed in 17 patients $(30.9 \%)$. The most common site of involvement was the gastrointestinal (GI) tract (25 patients, $45.5 \%$ ) followed by the lung (40\%), Waldeyer's ring (WR) (27.3\%), and ocular area (25.5\%). Ocular MZLs were commonly accompanied with WR- or lung-MZLs. GI-MZLs were WR or GI-MZLs. Lung-MZLs were frequently observed with ocular and GI-MZLs. WRMZLs were ocular or GI-MZLs. A total of 53 patients were treated, and 2 on watchful wait. As much as 48 patients received chemotherapy-based treatment. Among them, CR or PR was achieved in 38 patients $(79.2 \%, 95 \%$ CI $67-91 \%)$. Median time to progression (TTP) was 2.3 years (95\% CI 1.4-3.2 years). Cause-specific overall survival (OS) did not reach the median value. The 5-year OS rate was $84.9 \%$. MM-MZLs tend to be an indolent disease, characterized by prolonged survival with frequent relapses. The majority of cases could be controlled effectively via chemotherapy-based treatment, and prolonged survival was achieved in those patients. The GI, lung, WR, and ocular area were commonly presented with other MALT site

\section{H. S. Lee}

Department of Internal Medicine, Kosin University Gospel Hospital, Pusan, South Korea

\section{G.-W. Lee}

Division of Hematology-Oncology, Department of Internal Medicine, College of Medicine, Gyeongsang National University, Jinju, Korea

\section{H. S. Eom}

Hematology-Oncology Clinic, Center for Specific Organs Cancer, National Cancer Center, Goyang-si, Korea

\section{J.-Y. Kwak}

Department of Internal Medicine, Chonbuk National University Medical School, Chonju, Korea

\section{W. S. Lee}

Department of Hemato/Oncology, Busan Paik Hospital, College of Medicine, Inje University, Pusan, South Korea

\section{S. Y. Oh}

Medical Research Center for Cancer Molecular Therapy, Dong-A University College of Medicine, Pusan, South Korea

\section{Suh $(\bowtie)$}

Department of Oncology, Asan Medical Center, University of Ulsan College of Medicine,

86 Asanbyeongwon-gil, Songpa-gu, Seoul 138-736, Korea e-mail: csuh@amc.seoul.kr
MZLs, and an organ-specific relationship appears to be relevant to MM-MZLs.

Keywords Multiple - Mucosa-associated lymphoid tissues $\cdot$ Marginal zone $\cdot$ B cell lymphoma

\section{Introduction}

Marginal zone lymphoma (MZL) is a distinct subgroup of non-Hodgkin's lymphoma (NHL), which is typically characterized by an indolent clinical course and prolonged survival duration [1-4]. MZL of the mucosa-associated lymphoid tissue (MALT) type has been shown to be responsible for approximately 7-8\% of all NHL. In Korea, MZL accounts for $17 \%$ of all B cell lymphomas, and is the second most frequent histologic subtype, after diffuse large B cell lymphoma [5].

Mucosa-associated lymphoid tissue lymphoma is usually confined to a single extranodal organ with the acquired tissue, and has a favorable prognosis [3, 6, 7]. Otherwise, patients who initially presented with multiple MALT organs involving MZL accounted for $13-32 \%$ of all MZL patients $[1,3,8,9]$. Although they were classified as stage IV MZL, if these cases were not accompanied by nodal or bone marrow involvement, the prognosis was as good as that of stage I or II cases [3].

There is controversy as to whether WR should be considered as a nodal or extranodal site. Waldeyer's ring (WR) is considered the point of contact between MALT and peripheral lymphoid tissue of the lymph node. In addition to having characteristics of peripheral lymphoid tissue of the lymph node, these lymphoid tissues show characteristics of MALT, such as the absence of sinusoids, but the presence of a marginal zone, direct contact with epithelium, and intraepithelial B-lymphocytes.

Certain relationships have been reported to exist between co-involved organs in the lymphoma-WR-gastrointestinal (GI) tract $[10,11]$. According to a previous study of MZL, as well as the observed contralateral organ involvement and involvement within the same tract in the orbital, salivary gland, lung, and intestinal groups (paired organs), specific dissemination patterns appear to be present and characteristic to the primary site of presentation, supporting the notion that the expressions of special homing receptors and adhesion molecules are involved in the trafficking of MALTspecific lymphocytes to MALT-containing organs [9]. However, owing to its rarity and the small sample numbers reviewed thus far, it is difficult to identify any specific relationships among involved organs, clinical features, and prognosis.

In this study, we conducted retrospective analyses of multiple MALT organs involving MZLs (MM-MZLs) 
in order to identify their clinical features, treatment, prognosis, and specific relationships among involved organs.

\section{Patients and methods}

\subsection{Patients and data}

Eligible patients for this retrospective analysis were initially diagnosed with MZL according to the REAL/WHO classification criteria. MM-MZL was defined as that MZL which involved synchronously more than 2 different MALT organs at the presentation. Multiple involvements within one MALT organ (e.g. both side ocular lesions, multiple lung nodules, and multiple stomach lesions, etc.) were excluded. Unified case report forms were provided to the participating institutions. The collected data included age, gender, performance status, stage according to the definitions of the Ann Arbor staging system, location of primary involvement, presence of B symptoms, hemoglobin, absolute lymphocyte count (ALC), lactic dehydrogenase (LDH), hepatitis serology, initial date of diagnosis, and treatment modality utilized. We also obtained data regarding time to relapse, relationship with primary site and relapsing site, salvage treatment modality, and response and survival rates after salvage treatment.

Retrospectively, this data collection of patients with multiple MALT sites involving MZL was approved by the local ethical committee.

\subsection{Histology}

Diagnoses of marginal zone B cell lymphoma were based on the characteristic histological findings established in the WHO classifications, as well as the results of immunohistochemical staining for CD20 and CD3. Cases which proved difficult to exclude other low-grade B cell lymphomas, immunohistochemical studies for CD5, CD10, CD23, cyclin D1, BCL6, and Ki-67 were conducted. Because MZL is commonly accompanied by reactive hyperplasia, gene rearrangement studies for the $\mathrm{IgH}$ gene were conducted via PCR analysis, in an attempt to exclude any benign hyperplasia.

\subsection{Statistical analysis}

The relatively high incidence rates related to specific organs were compared by $t$ tests. Categorical variables in the two groups were compared via $\chi^{2}$ or Fisher's exact tests. $P$ values $<0.05$ were considered statistically significant, and all $P$ values corresponded to two-sided significance tests. Overall survival (OS) and time to progression (TTP) were estimated via the Kaplan-Meier product-limit method. TTP was calculated from the date on which treatment began after relapse to the date on which the disease progression was recognized or the date of the final follow-up visit. OS was measured from the date of relapse to the date of death or the date of the final follow-up visit. Survival rates were compared for statistical differences via log-rank analysis, and Cox's regression model was utilized for multivariate analysis at a $P$ value of $<0.05$ in the univariate (by log-rank test) analysis of OS and TTP. All data were analyzed using SPSS software (Version 18.0, Chicago, IL, USA).

\section{Results}

\subsection{Patient characteristics}

Between October 1995 and February 2009, a total of 55 patients who were diagnosed histologically with MMMZLs from 17 different institutions in Korea were included in this study.

The clinical presentations of the patients initially diagnosed with MZL are provided in Table 1.

The numbers of males and females in this study were 41 and 14, respectively. The median age was 59 years (range 30-82 years). The majority of patients $(85.5 \%)$ evidenced good performance status. B symptoms were noted in 10 patients. Lymph node (LN) involvement was noted in almost all of the patients $(89.1 \%)$. BM involvement was detected in 17 patients $(30.9 \%$ ) (Table 2). As much as $45.5 \%$ of the patients (25 of 55 ) were categorized into the low or low-intermediate risk groups, in accordance with the International Prognostic Index (IPI). According to the Marginal Zone Lymphoma Prognostic Index (MZLPI) [7], $85.5 \%$ (47 of 55) were assigned to the low-risk group. The numbers of involved organs $(2,3$, and 4$)$ were $38(69.1 \%)$, $13(23.6 \%)$, and $4(7.3 \%)$, respectively. The most frequent sites of involvement were the GI tract $(45.5 \%)$, followed by the lung (40\%), WR (27.3\%), and tonsil (25.5\%) (Table 3).

\subsection{Response to treatments}

A total of 55 patients were treated, and 2 were observed until progression on watchful wait status (Table 4). Among 53 patients, 48 received chemotherapy-based treatments. Among those patients, CR or PR was achieved in 38 (79.2\%; 95\% CI 67-91\%). Treatment with only chemotherapy without operation or radiotherapy was administered to 34 patients. A combination of rituximab and chemotherapy or rituximab applied singly was utilized in the treatment of 23 patients. Except for 1 patient, all of the patients enjoyed clinical benefits (Table 5). 
Table 1 Patients' characteristics

\begin{tabular}{|c|c|c|}
\hline & $N=55$ & $\%$ \\
\hline \multicolumn{3}{|l|}{ Gender } \\
\hline Male & 41 & 74.5 \\
\hline Female & 14 & 25.5 \\
\hline \multicolumn{3}{|l|}{ Age } \\
\hline Median & $59(30-82)$ & \\
\hline$\geq 60$ & 27 & 49.1 \\
\hline$<60$ & 28 & 50.9 \\
\hline \multicolumn{3}{|c|}{ Lactic dehydrogenase (LDH) } \\
\hline Normal & 34 & 61.8 \\
\hline$\geq$ Normal & 14 & 25.5 \\
\hline Unchecked & 7 & 12.7 \\
\hline \multicolumn{3}{|l|}{ Hemoglobin } \\
\hline$\geq 12 \mathrm{~g} / \mathrm{dL}$ & 31 & 56.4 \\
\hline$<12 \mathrm{~g} / \mathrm{dL}$ & 18 & 32.7 \\
\hline Unreported & 6 & 10.9 \\
\hline \multicolumn{3}{|l|}{ ANC } \\
\hline$\geq 1.0 \times 10^{9} / \mathrm{L}$ & 38 & 69.1 \\
\hline$<1.0 \times 10^{9} / \mathrm{L}$ & 10 & 18.2 \\
\hline Unreported & 7 & 12.7 \\
\hline \multicolumn{3}{|l|}{ PS (ECOG) } \\
\hline $0-1$ & 47 & 85.5 \\
\hline 2 & 8 & 14.5 \\
\hline \multicolumn{3}{|l|}{ B symptom } \\
\hline A & 45 & 81.8 \\
\hline $\mathrm{B}$ & 10 & 18.2 \\
\hline \multicolumn{3}{|c|}{ No. of involving organs } \\
\hline 2 & 38 & 69.1 \\
\hline 3 & 13 & 23.6 \\
\hline 4 & 4 & 7.3 \\
\hline \multicolumn{3}{|l|}{ IPI } \\
\hline Low & 4 & 7.3 \\
\hline Low-intermediate & 21 & 38.2 \\
\hline High-intermediate & 19 & 34.5 \\
\hline High & 8 & 14.5 \\
\hline Undetermined & 3 & 5.5 \\
\hline \multicolumn{3}{|l|}{ MZLPI } \\
\hline Intermediate & 47 & 85.5 \\
\hline High & 8 & 14.5 \\
\hline
\end{tabular}

$\overline{L D H}$ lactic dehydrogenase, ANC absolute lymphocyte count, $P S$ $(E C O G)$ Eastern Cooperative Oncology Group Performance Status, IPI international prognostic index, MZLPI marginal zone B cell lymphoma prognostic index

In the cases of MM-MZL involving nearby organs or operable lesions, local radiotherapy or operation treatments were performed on 5 patients. CR was achieved in all 5 of those patients. Three patients transformed DLBCL during follow-up.
Table 2 Extents of dissemination

\begin{tabular}{lrr}
\hline & $N=55$ & $\%$ \\
\hline Multi-sited marginal zone B cell lymphoma & & \\
Alone & 9 & 16.4 \\
+Lymph node & 25 & 45.5 \\
+Bone marrow & 6 & 10.9 \\
+Bone marrow + Lymph node & 11 & 20.0 \\
+Liver + Lymph node & 4 & 7.3 \\
\hline
\end{tabular}

Table 3 Organ distributions of multiple organs involving marginal zone B cell lymphoma

\begin{tabular}{|c|c|c|c|c|}
\hline Sites & OCU & WR & LUNG & GI \\
\hline OCU & & 4 & 5 & 2 \\
\hline PNS & & & 1 & \\
\hline $\mathrm{NC}$ & 1 & & & \\
\hline $\mathrm{OC}$ & 1 & 2 & & 2 \\
\hline Larynx & & 1 & & \\
\hline WR & 4 & & & 8 \\
\hline Salivary & & 2 & & 1 \\
\hline Lung & 5 & & & 10 \\
\hline Pericardium & & & 1 & \\
\hline GI & 2 & 8 & 10 & \\
\hline Peritoneum & & & 2 & 1 \\
\hline Spleen & & 2 & 2 & 2 \\
\hline Kidney & 1 & & 1 & \\
\hline Adrenal & & & 2 & 1 \\
\hline Bladder & & & 1 & \\
\hline Prostate & & & 2 & \\
\hline Bone & & & 1 & \\
\hline Muscle & 2 & & 1 & \\
\hline Skin & & 1 & 1 & 1 \\
\hline No. of patients & 14 & 15 & 22 & 25 \\
\hline
\end{tabular}

$O C U$ ocular and adnexa, $P N S$ paranasal sinus, $N C$ nasal cavity, $O C$ oral cavity, WR Waldeyer's ring, GI gastrointestinal tract

\subsection{TTP and OS of multiple MALT organs involving MZL}

Median TTP was 2.3 years (95\% CI 1.4-3.2 years) (Fig. 1). OS did not reach the median value. The 5-year OS rate was 84.9\%. Gender, age, LN involvement, BM involvement, ALC, LDH, performance status, and the use of rituximab or anthracycline were analyzed for evaluations of prognostic value. However, no relevant predictive clinical factor was detected for reduced TTP and OS for MM-MZL.

\subsection{Organ involvement relationship of multiple MALT organs involving MZL}

The most common site of involvement was the GI tract (25 patients, $45.5 \%$ ) followed by the lung (40\%), WR (27.3\%), and the ocular area $(25.5 \%)$. 
Table 4 Treatment result of multiple organs involving marginal zone B cell lymphoma

\begin{tabular}{|c|c|c|c|c|c|c|}
\hline & $N=55$ & $\mathrm{CR}(\%)$ & PR $(\%)$ & $\mathrm{SD}(\%)$ & $\mathrm{PD}(\%)$ & NE $(\%)$ \\
\hline \multicolumn{7}{|l|}{ Chemotherapy } \\
\hline Alone & 34 & $17(50.0)$ & $8(23.5)$ & 7 (20.6) & $1(2.9)$ & $1(2.9)$ \\
\hline +Operation & 7 & $6(85.7)$ & $1(14.3)$ & & & \\
\hline +Radiotherapy & 4 & $1(25.0)$ & $3(75.0)$ & & & \\
\hline+ Operation + radiotherapy & 3 & $1(33.3)$ & $1(33.3)$ & & $1(33.3)$ & \\
\hline Radiotherapy & 3 & $3(100)$ & & & & \\
\hline Operation & 2 & $2(100)$ & & & & \\
\hline Observation & 2 & & & $2(100)$ & & \\
\hline
\end{tabular}

$C R$ complete response, $P R$ partial response, $S D$ stable disease, $P D$ progression of disease, $N E$ not evaluated

Table 5 Chemotherapy result of multiple organs involving marginal zone B cell lymphoma

\begin{tabular}{lcllll}
\hline Chemotherapy & $N=34$ & CR $(\%)$ & PR (\%) & SD (\%) & PD (\%) \\
\hline R-CVP & 16 & $8(50)$ & $4(25)$ & $3(18.8)$ & $1(6.2)$ \\
CHOP & 6 & $1(16.7)$ & $3(50)$ & $2(33.3)$ & \\
CVP & 5 & $3(60)$ & & $2(40)$ & \\
R-CHOP & 5 & $4(80)$ & $1(20)$ & & \\
Z-RCVP & 1 & $1(100)$ & & & \\
R & 1 & & & $1(100)$ &
\end{tabular}

$C R$ complete response, $P R$ partial response, $S D$ stable disease, $P D$ progression of disease

CHOP Cyclophosphamide + Doxorubicin, + Vincristine + Prednisone, $(R)-C V P$ (Rituximab)-Cyclophosphamide + Vincristine + Prednisone, Z-RCVP R-CVP followed by zevalin

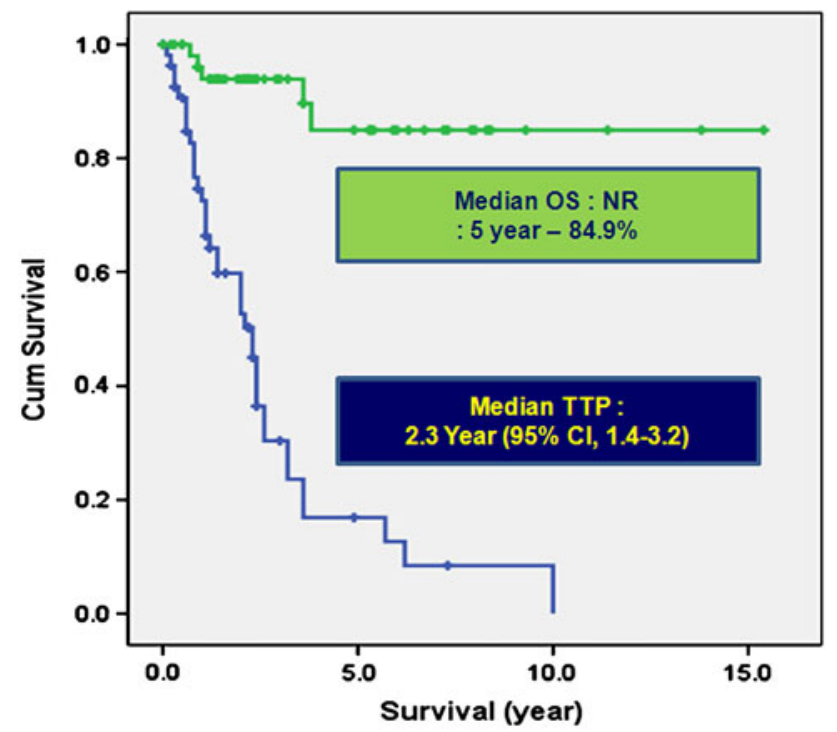

Fig. 1 Median time to progression (TTP) was 2.3 years (95\% CI $1.4-3.2$ years). The 5-year overall survival (OS) rate was $84.9 \%$. NR not reached
A total of 14 patients had combined ocular and adnexal MZL with MZL of other MALT organs. WR (4 patients) and lung (5 patient) were the most frequently co-involved organs ( $P=0.040$ and 0.019 , respectively). As much as 15 patients with WR-MZL also more frequently evidenced accompanying ocular and GI-MZL (4 and 8 patients) than was observed in other sites $(P=0.040$ and 0.019 , respectively). Patients who evidenced combined MZL of the lung with other MALT organs were 22. The most frequently involved MALT sites were ocular (5 patients, $P=0.021)$ and GI-MZL (10 patient, $P=0.001)$. The GI tract was the most commonly involved site of MM-MZL. A total of 25 patients evidenced co-involvement with GI-MZL. WR (8 patients, $P=0.003)$ and lung (10 patients, $P=0.001)$ were more frequently observed than other MALT sites (Fig. 2a-d).

\section{Discussion}

Mucosa-associated lymphoid tissue lymphoma is generally confined to a single extranodal organ. Advanced-stage MZL accounts for $20-50 \%$ of all MZL [1, 3, 8, 12], and MM-MZL accounts for approximately one-third of cases of advanced-stage MZL [1, 3, 8, 9]. In a study in which 158 patients were enrolled, only 18 (11\%) evidenced multiple organ localization [1]; 7 (3.5\%) exhibited stomach and intestinal tract involvement; and 5 (3\%) evidenced GI tract and lung involvement. In another recent retrospective survey of the International Extranodal Lymphoma Study Group in which 180 patients were analyzed, multiple MALT organ localizations were noted at diagnosis in $13 \%$ of all patients [3].

This pathogenesis of MM-MZLs has been previously explained with the results of characteristic expression of homing receptor or adhesion molecules on the surfaces of B cells in cases of MALT [9, 13]. Expression of mucosal lymphocytes and mucosal vascular addressin cell adhesion molecule-1 (MAdCAM-1) on high 
Fig. 2 a Ocular and adnexal involving multiple organs involving marginal zone $\mathrm{B}$ cell lymphoma (MM-MZ).

Waldeyer's ring (WR) (4 patients) and lung (5 patient) were the most frequently coinvolved organ $(P=0.040$ and 0.019). b WR- involving MM-

MZL. Ocular and

gastrointestinal tract (GI) MZL (4 patients and 8 patients) than was observed in other sites $(P=0.040$ and 0.019$)$. c Lung involving MM-MZL. The most frequently involved MALT sites were ocular (5 patients, $P=0.021)$ and GI-MZL (10 patients, $P=0.001)$. d The GI tract involving MM-MZL. WR (8 patients, $P=0.003$ ) and lung (10 patients, $P=0.001$ ) were more frequently observed than other MALT sites. PNS paranasal sinus a

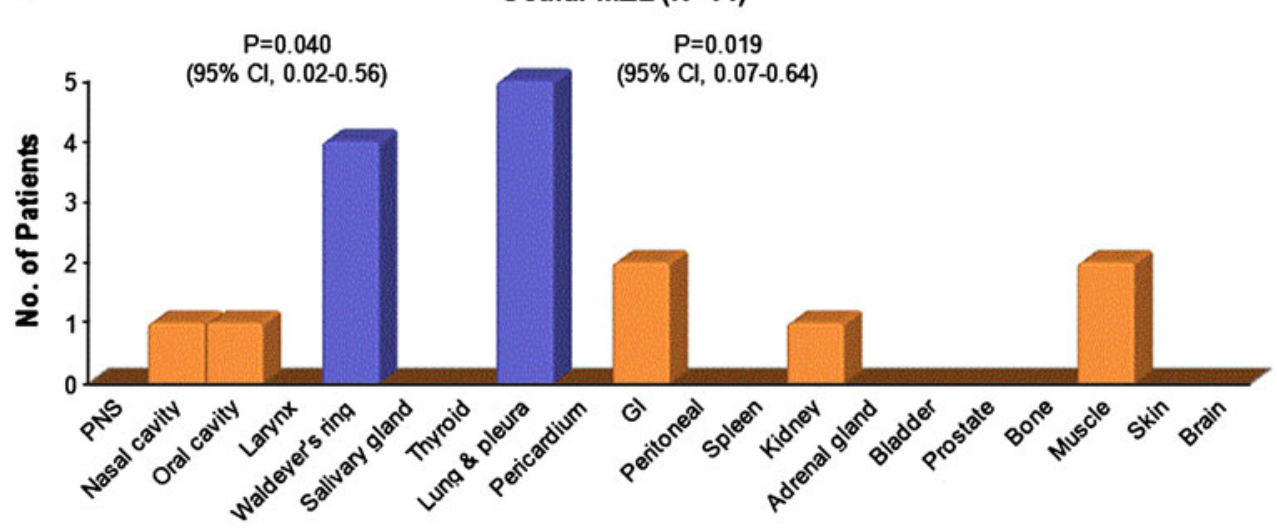

b

Waldeyer's ring MZL $(\mathrm{N}=15)$

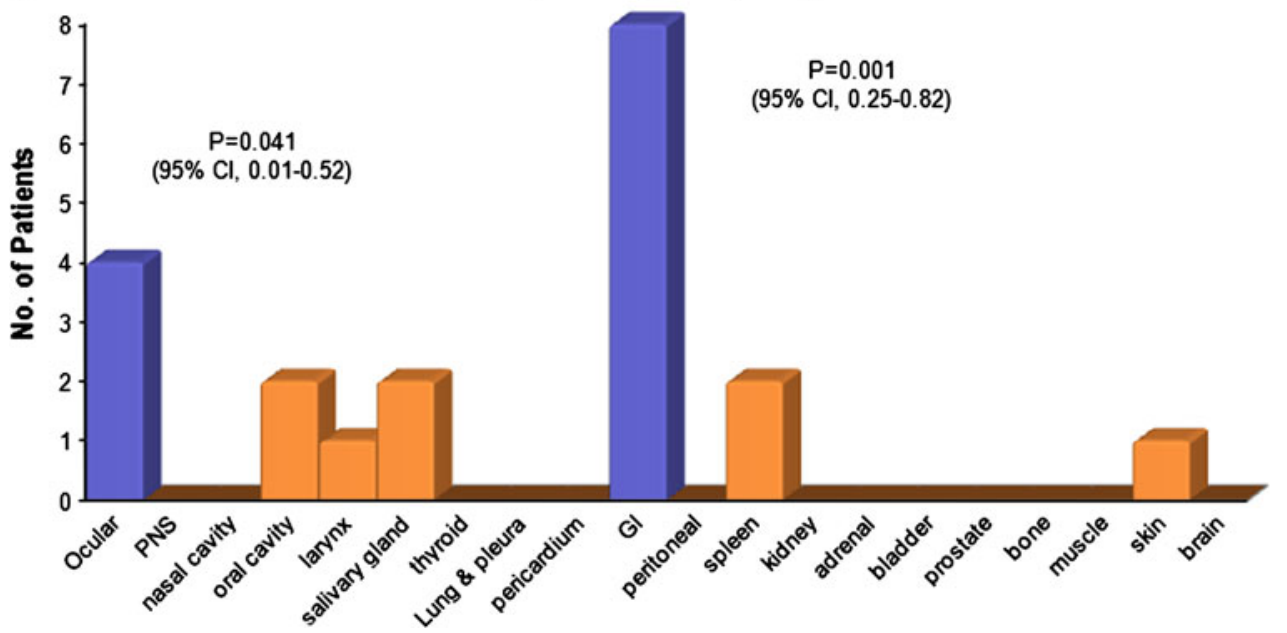

endothelial venules (HEV) may play an important role in lymphocyte migration at sites of chronic inflammation or MALT lymphoma of the gastrointestinal tract and thyroid. In other organs, peripheral lymph node vascular addressin (PNAd) may have the greater role [14]. And also $\alpha 4 \beta 7$ integrin and L-selectin may play an important role in the lymphocyte homing of gastrointestinal lowgrade MALT lymphoma [15]. But there is a controversy whether MM-MZL has clonal relationship or not [16, 17].

Paired organs (lung, orbit) generally exhibit a tendency toward bilateral involvement or toward being sites of relapse [6, 9]. However, inter-organ relationships have been reported in a limited series. In the aforementioned large-scale retrospective study, pulmonary MZL has been reported to exhibit a specific relationship with GI tract involvement [9]. Gastric localizations were diagnosed at staging gastroduodenoscopy and histologically confirmed (odds ratio for simultaneous gastric localization in the lung group was 14.3 ; 95\% CI 3.8-54.4, $p<0.0001$ ).

In our survey, multiple mucosal site involvement concerned not only the GI tract, with dissemination to the stomach or intestines, but also the GI tract and non-GI tract organs, along with dissemination to other mucosal organs. Although GI tract and lung involvement in MM-MZL was the most common type, this relationship was also noted in GI and WR MM-MZL with a similar pattern, as in the cases of NHL.

Moreover, between the non-GI tract organs, WR-ocular and ocular-lung, a relatively higher incidence of coappearance was noted. The relationship between non-GI tract organ and MZL has yet to be reported. This may originate from the rare incidence of MZL, allowing for the relationships between two organs to be characterized. If we synthesize our analysis, we can draw a relational figure between involved MALT organs (Fig. 3). Interestingly, no ocular GI tract and WR-lung were noted. According to a previous prospective analysis, secondary gastric spread was virtually absent in parotid and orbital lymphoma; this finding is consistent with our data [18]. Therefore, they suggested that an extensive GI work-up in the absence of clinical symptoms does not appear strictly necessary in patients diagnosed with MALT lymphoma of the ocular adnexa. 
Fig. 2 continued
C

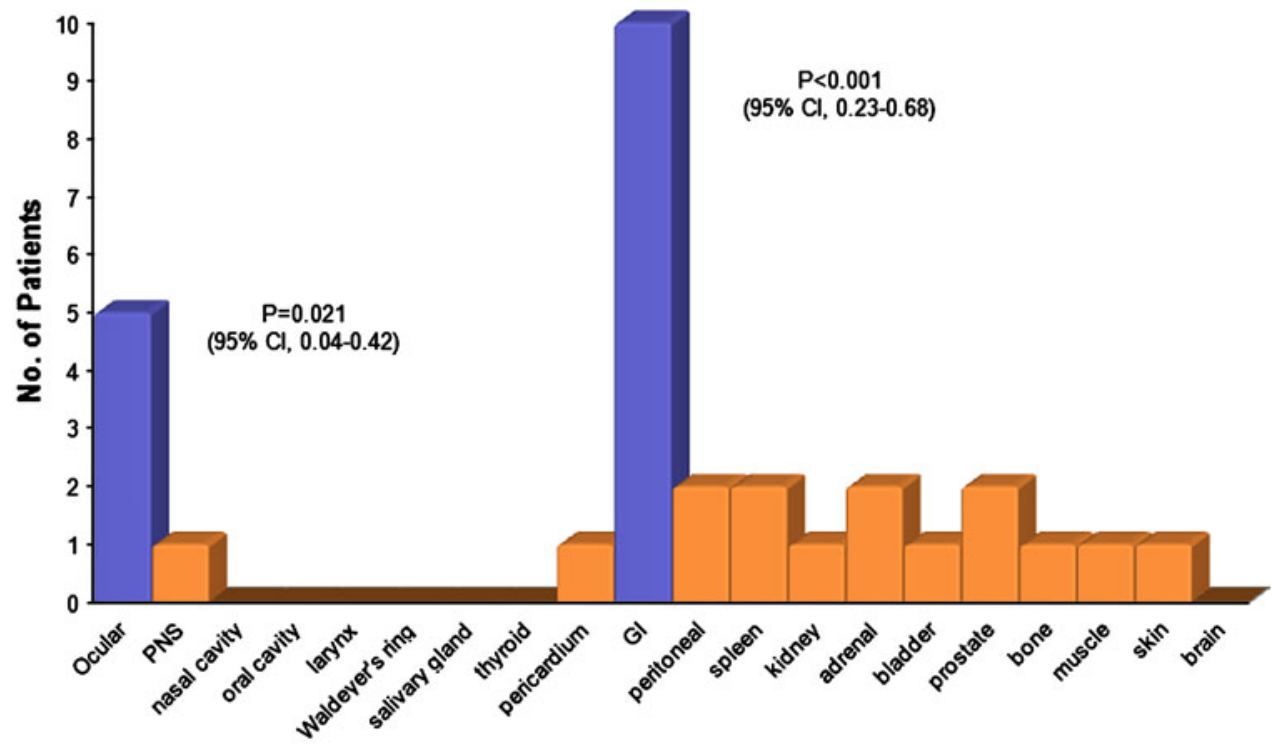

d

Gastrointestinal MZL $(\mathrm{N}=\mathbf{2 5})$

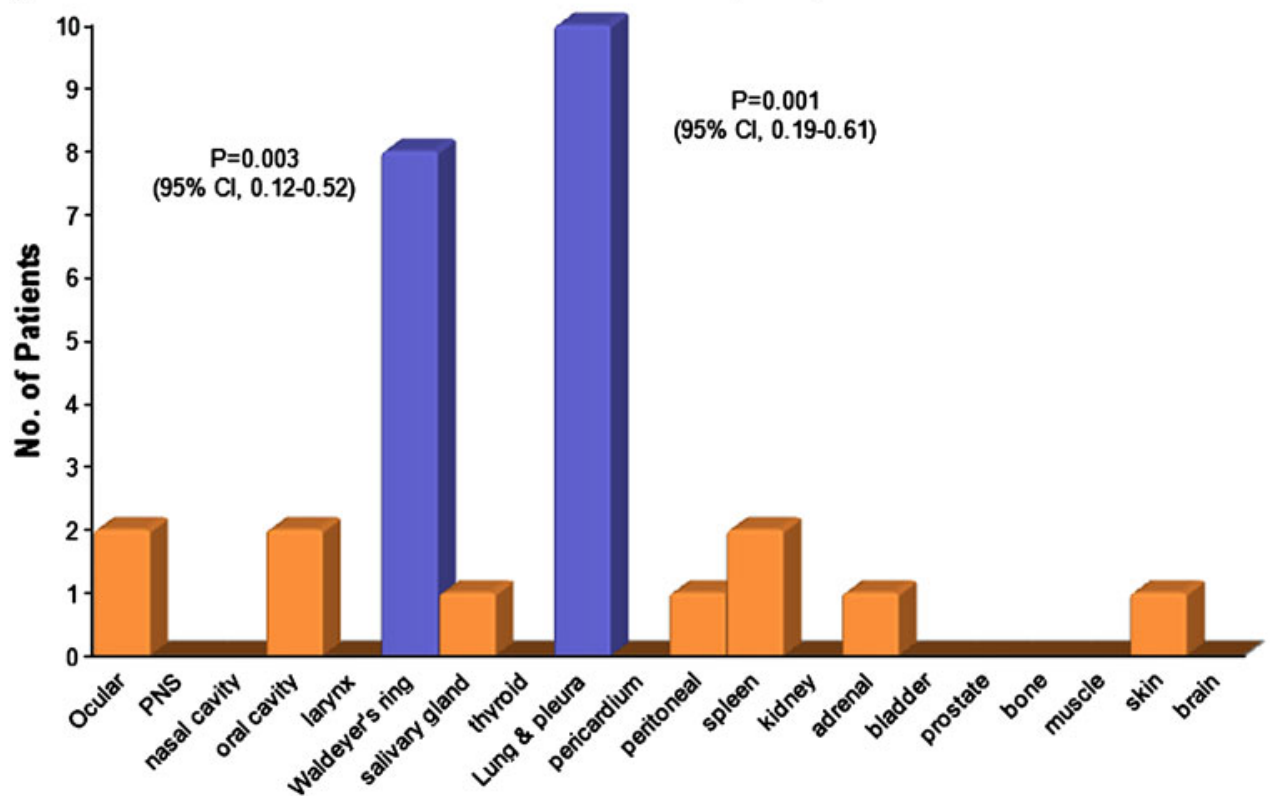

In previous prospective and retrospective studies, gastric and extragastric MZL have been shown to evidence only minimally different dissemination patterns and prognoses $[9,18]$. In cases of gastric MZL, almost $50 \%$ of patients evidenced dissemination within the GI tract. Extragastric MALT lymphomas are significantly more likely to disseminate than gastric MALT lymphomas and evidence shorter duration in TTP. In our analysis, we did not separate gastric organs from extra-gastric organs; thus, it was actually impossible to determine which site is the primary site in MMMZL. Gastric organ- and extra-gastric organ-involved MMMZLs were also analyzed for TTP and OS. However, our data showed no differences in TTP and OS duration. Other proposed factors-regional LN involvement, BM involvement, use of rituximab, and anthracycline and advanced age-were not prognostically significant.

This study was undergone based on the retrospective medical record review. Therefore, it could have a lot of bias, such as selected patient data collection, insufficient staging work-up, different treatment regimens, and loss of follow-up. Staging work-up and treatment modalities were all carried out in accordance with the institution's recommended protocols. Chest computed tomography (CT), Abdomino-pelvic CT, involved area CT, BM examination, 


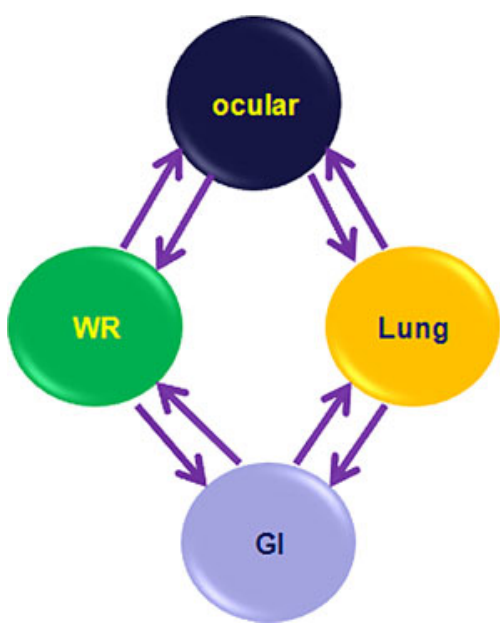

Fig. 3 The scheme of multiple organs involving marginal zone B cell lymphomas. WR Waldeyer's ring, GI gastrointestinal tract, Ocular orbit and ocular adnexa

and physical evaluation for palpable $\mathrm{LN}$ were recommended to evaluate disease status. In cases involving Waldeyer's ring, GI study was recommended. But, all the recommendation did not perform to every MZL patients. In this study, except 1 patient, all patients had performed the BM evaluation. But, 12 out of 15 Waldeyer's ring MZL patients had performed GI tract evaluation (endoscopy and colonoscopy). Therefore, a little percentage of incidences might have a possibility of underestimation. Additionally, we did not take genetic factors into consideration. According to the findings of a previous study, multifocality was significantly associated with $t(11 ; 18)(\mathrm{q} 21 ; \mathrm{q} 21)$ in gastric lymphomas and with trisomy 18 in extra-gastric lymphomas [18]. This could have generated bias in the results and conclusions of our data analysis.

In conclusion, the results of our survey show that MMMZL tends to be an indolent disease-it is characterized by prolonged survival with frequent relapses, similar to MZL involving other single MALT sites. The majority were controlled well with chemotherapy-based treatments, allowing for prolonged survival durations. However, no prognostic factors of MM-MZL in the GI, lung, WR, and ocular area were commonly detected with other MALT-site MZL; this appears to be an organ relationship that commonly exists in MM-MZLs.

Acknowledgments This Paper was supported by the Dong-A University Research Fund. This work was supported by the Korea Science and Engineering Foundation (KOSEF) grant funded by the Korea government (MEST; R13-2002-044-05001-0).

\section{References}

1. Thieblemont C, Berger F, Dumontet C, Moullet I, Bouafia F, Felman P, et al. Mucosa-associated lymphoid tissue lymphoma is a disseminated disease in one-third of 158 patients analyzed. Blood. 2000;95:802-6.

2. Zinzani PL, Magagnoli M, Galieni P, Martelli M, Poletti V, Zaja $F$, et al. Nongastrointestinal low-grade mucosa-associated lymphoid tissue lymphoma: analysis of 75 patients. J Clin Oncol. 1999; $17: 1254$

3. Zucca E, Conconi A, Pedrinis E, Cortelazzo S, Motta T, Gospodarowicz MK, et al. Nongastric marginal zone B-cell lymphoma of mucosa-associated lymphoid tissue. Blood. 2003;101:2489-95.

4. Isaacson PG. Update on MALT lymphomas. Best Pract Res Clin Haematol. 2005;18:57-68.

5. Ko YH, Kim CW, Park CS, Jang HK, Lee SS, Kim SH, et al. REAL classification of malignant lymphomas in the Republic of Korea: incidence of recently recognized entities and changes in clinicopathologic features. Hematolymphoreticular Study Group of the Korean Society of Pathologists. Revised EuropeanAmerican lymphoma. Cancer. 1998;83:806-12.

6. Oh SY, Kwon HC, Kim WS, Hwang IG, Park YH, Kim K, et al. Intestinal marginal zone B-cell lymphoma of MALT type: clinical manifestation and outcome of a rare disease. Eur J Haematol. 2007;79:287-91.

7. Oh SY, Kwon HC, Kim WS, Park YH, Kim K, Kim HJ, et al. Nongastric marginal zone B-cell lymphoma: a prognostic model from a retrospective multicenter study. Cancer Lett. 2007;258:90-7.

8. Kalpadakis C, Pangalis GA, Vassilakopoulos TP, Kyrtsonis MC, Siakantaris MP, Kontopidou FN, et al. Non-gastric extra-nodal marginal zone lymphomas-a single centre experience on 76 patients. Leuk Lymphoma. 2008;49:2308-15.

9. de Boer JP, Hiddink RF, Raderer M, Antonini N, Aleman BM, Boot H. Dissemination patterns in non-gastric MALT lymphoma. Haematologica. 2008;93:201-6.

10. Ree HJ, Rege VB, Knisley RE, Thayer WR, D'Amico RP, Song JY, et al. Malignant lymphoma of Waldeyer's ring following gastrointestinal lymphoma. Cancer. 1980;46:1528-35.

11. Gospodarowicz MK, Sutcliffe SB, Brown TC, Chua T. Bush RS Patterns of disease in localized extranodal lymphomas. J Clin Oncol. 1987;5:875-80.

12. Oh SY, Ryoo BY, Kim WS, Park YH, Kim K, Kim HJ, et al. Nongastric marginal zone B-cell lymphoma: analysis of 247 cases. Am J Hematol. 2007;82:446-52.

13. Briskin M, Winsor-Hines D, Shyjan A, Cochran N, Bloom S, Wilson $\mathrm{J}$, et al. Human mucosal addressin cell adhesion molecule-1 is preferentially expressed in intestinal tract and associated lymphoid tissue. Am J Pathol. 1997;151:97-110.

14. Liu Y-X, Ohara N, Yoshino T, Jin G-S, Akagi T. Expression of MAdCAM-1 and PNAd in inflammatory and MALT lymphoma tissues of ocular adnexa, thyroid. Salivary gland and lung. J Clin Exp Hematopathol. 2004;44:33-7.

15. Liu YX, Yoshino T, Ohara N, Oka T, Jin ZS, Hayashi K, et al. Loss of expression of alpha4beta7 integrin and L-selectin is associated with high-grade progression of low-grade MALT lymphoma. Mod Pathol. 2001;14:798-805.

16. Konoplev S, Lin P, Qiu X, Medeiros LJ. Yin CC Clonal relationship of extranodal marginal zone lymphomas of mucosaassociated lymphoid tissue involving different sites. Am J Clin Pathol. 2010;134:112-8.

17. Yoshino T, Ichimura K, Mannami T, Takase S, Ohara N, Okada $\mathrm{H}$, et al. Multiple organ mucosa-associated lymphoid tissue lymphomas often involve the intestine. Cancer. 2001;91:346-53.

18. Raderer M, Wohrer S, Streubel B, Troch M, Turetschek K, Jager $\mathrm{U}$, et al. Assessment of disease dissemination in gastric compared with extragastric mucosa-associated lymphoid tissue lymphoma using extensive staging: a single-center experience. J Clin Oncol. 2006;24:3136-41. 